\title{
AN IMPROVED PERTURB AND OBSERVE MPPT ALGORITHM WITH VARIABLE
} STEP

\author{
S. POPESCU \\ BOC Group, Operngasse 20b, Vienna, Austria \\ E-mail: sabin.popescu@boc-group.com
}

\begin{abstract}
The algorithm for maximum power point tracking (MPPT) using a fixed is widely used because of its simplicity and easyness to implement. This paper presents an improvement consisting in a variable step (VS) applied to the standard Hill Climbing MPPT technique to improve both accuracy and tracking speed. Drawbacks will still be encountered in VS tracking algorithms, between response time and power oscillation.
\end{abstract}

Keywords: photovoltaic system, MPPT, optimization method

\section{INTRODUCTION}

Because of the finite nature of conventional energy sources, increased oil and gas prices since the 1970's and the need of cleaner energy, more and more countries are accepting the true potential of renewable energy sources. The most widely used renewable energy resources are solar, wind and hydro. Of these, the most suitable for generating massive amounts of power is solar energy, mostly because of its better predictibility among the above.

A photovoltaic (PV) module has nonlinear I-V and P-V characteristics and the output of the PV module varies with changes in the weather conditions, the most important being solar irradiance and temperature. For being able to tap the PV power source close to its full potential, an MPPT algorithm needs to be used. Several techniques can currently be used for designing an MPPT algorithm [1], but among these, the most widely used are the Perturb and Observe (PnO) or Hill-Climbing and INC MPPT algorithm. PnO provides higher speed, but oscillates around the maximum power point (MPP), whilst INC doesn't oscillate, but tends to reach the MPP slower than PnO [2]. INC is used very often to reduce the downside of the $\mathrm{PnO}$ algorithm, with the main advantage being its increased stability under rapidly changing weather conditions [3]. However, the conventional INC algorithm which uses a perturbation with a fixed step size will also produce oscillations around the Maximum Power Point (MPP).

This paper proposes a modified $\mathrm{PnO}$ algorithm, that uses a variable step (VS), to reduce the trade-off problem between the response time of the system and power oscillation after having reached the MPP.

\section{MODELING THE PV GENERATOR}

PV generators consist of multiple PV modules connected in parallel and series for a given operating voltage and output power [5]. The models of the PV generators are deduced from the models of solar cells; several studies propose using one diode or two diodes (more precise) models. In this paper we use the conventional single diode model presented in Figure 1.

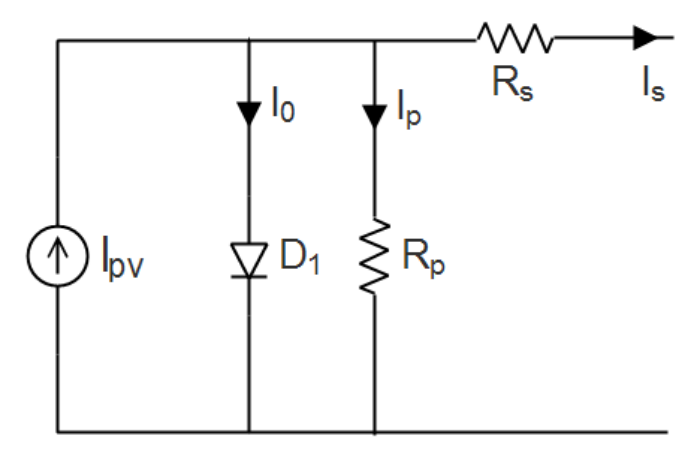

Figure 1. Conventional Single Diode Model of the PV Cell

$\mathrm{I}_{\mathrm{pv}}$ is the photogenerated current based on the irradiance level, Id the diode current, $\mathrm{R}_{\mathrm{p}}$ and $\mathrm{R}_{\mathrm{s}}$ are the parallel (shunt) and series resistances, respectively. Based on Figure 1, the dependence between the output voltage and current can be written as:

$$
I_{S}=I_{p v}-I_{0}\left[e^{\frac{q\left(V_{p v}+I_{S} R_{S}\right)}{K T A}}-1\right]-\left(\frac{V_{p v}+I_{S} R_{S}}{R_{P}}\right)
$$

where $I_{0}$ is the dark current.

The model presented in Figure 1, has been used for simulating the PV cell in pSpice. This way we have identified the influence of the operating temperature (Figure 2) and the effect of the solar radiance on the MPPT (Figure 3). 


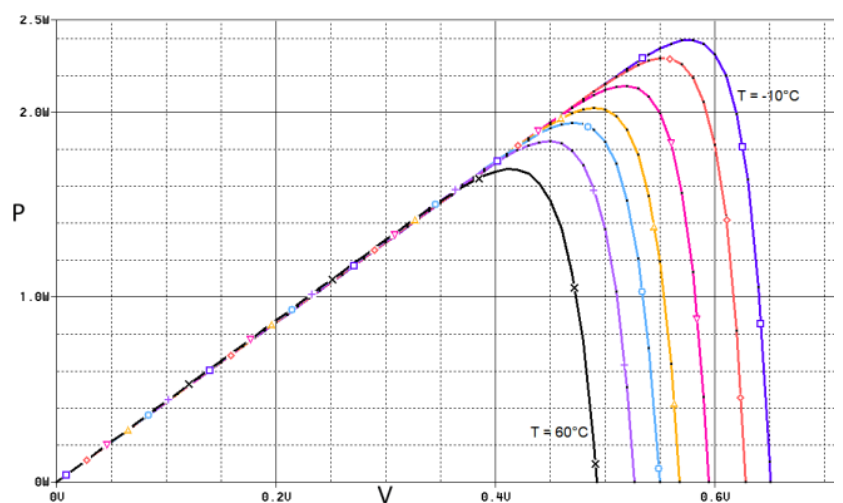

Figure 2. Temperature effect on the P-V curve

The Maximum Power Point varies very little when radiation levels are singnificantly changing, but a change in ambient temperature has a big impact on the MPP. On the other hand, the maximum power decreases significantly when the radiation levels drop, but only by a small margin when the ambient temperature rises.

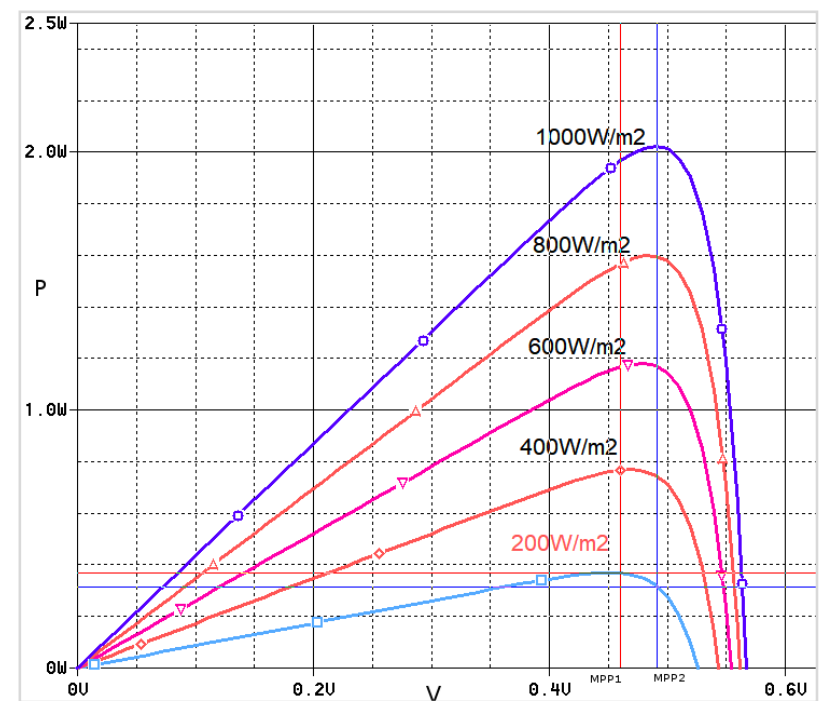

Figure 3. Irradiance dependence of PV characteristics

Tracking the MPP is not a simple task, esentially because the PV generator P-V and I-V curves depend on both operating temperature and incident radiance, which in turn can change very fast over short periods of time.

\section{TRACKING THE MAXIMUM POWER POINT}

Over the years, several methods have been proposed for tracking maximum power points for PV arrays [6-8], but the most used one seems to be Perturb and Observer $(\mathrm{PnO})$. This is an iterative method, that perturbs the working photovoltage $\mathrm{V}_{\mathrm{pv}}$ and analyses the the resulted power compared to the previous one. Figure 4 describes the way the PnO method works.

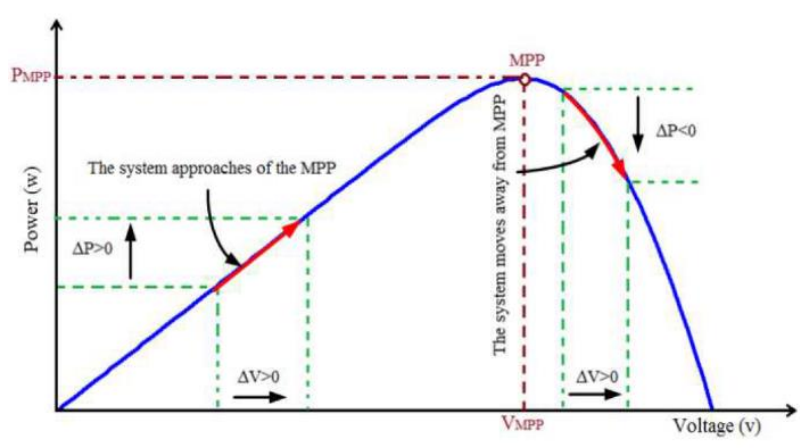

Figure 4. The functioning principle of the PnO method

If, for an increment in $\mathrm{Vpv}$, the output power $\Delta \mathrm{Ps}$ higher than zero $(\mathrm{Vpv}>0)$, the system is moving towards the maximum power point; if $\Delta \mathrm{P}$ is negative, then the system is moving away of the MPP. Figure 5 depicts the flow chart of the $\mathrm{PnO}$ algorithm.

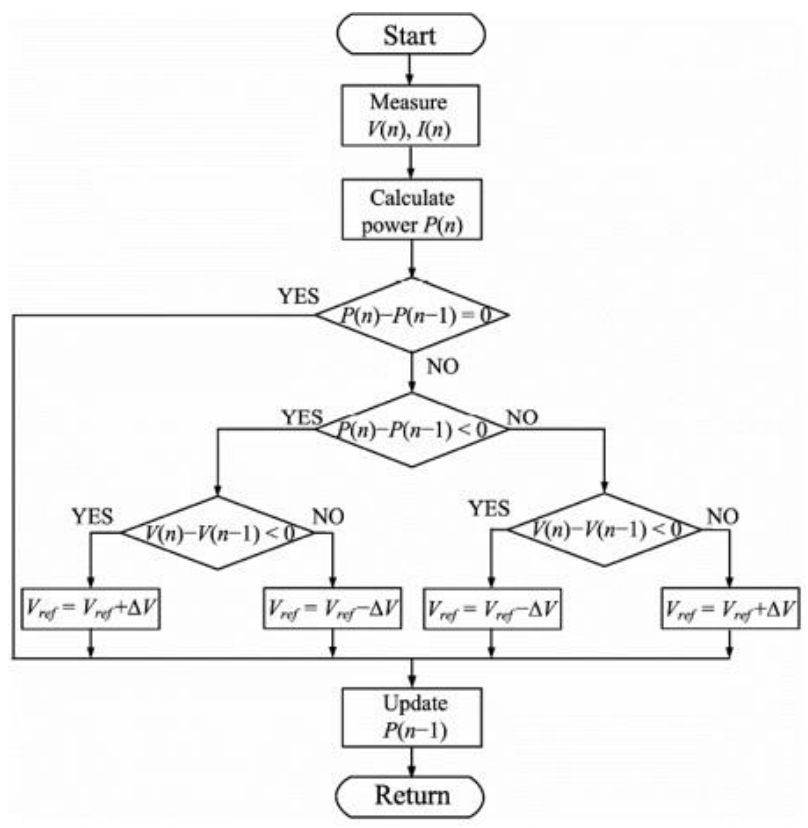

Figure 5. Flow Chart of PnO Algorithm

Another very often used iterative method for tracking the MPP is the Incremental Conductance Method (INC), which has a principle similar to $\mathrm{PnO}$, but instead of perturbing the photovoltage and analysing the resulting Power, it observes the variation of the conductance. The flow chart of the INC algorithm is depicted in the Figure 6. 


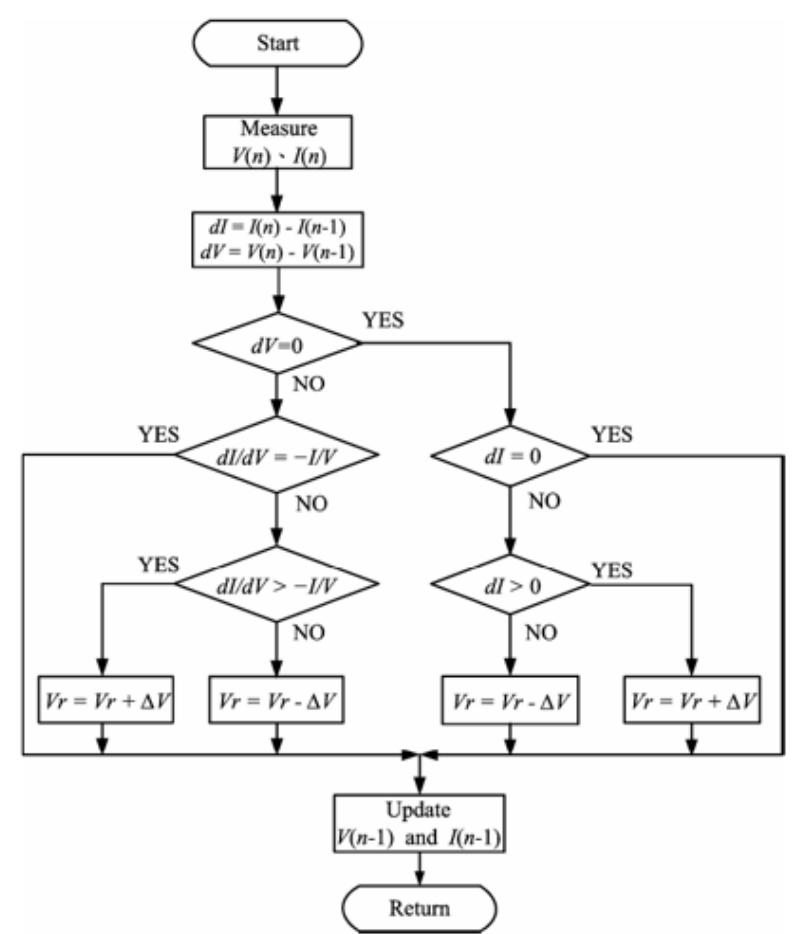

Figure 6. Flow Chart of the INC Method

The PnO algorithm has a better response time, reaching the MPP much faster than the INC algorithm, but oscillates around it, thus losing efficiency. The INC algorithm reaches the MPP slower, but the absence of oscillations gives it better performance when operating under stable weather conditions: temperature and radiance.

In order to eliminate the trade-off between response time and efficiency loss due to oscillations, an improved $\mathrm{PnO}$ algorithm is proposed, where the step used to perturb the photovoltage is not constant, but varies proportional to the slope of the P-V characteristic.

\section{SIMULATION RESULTS AND DISCUSSION}

We have used MatLab and SimuLink for modelling the PV system and simulating the three MPPT algorithms under various changes of the weather conditions. The SimuLink model is presented in Figure 7.

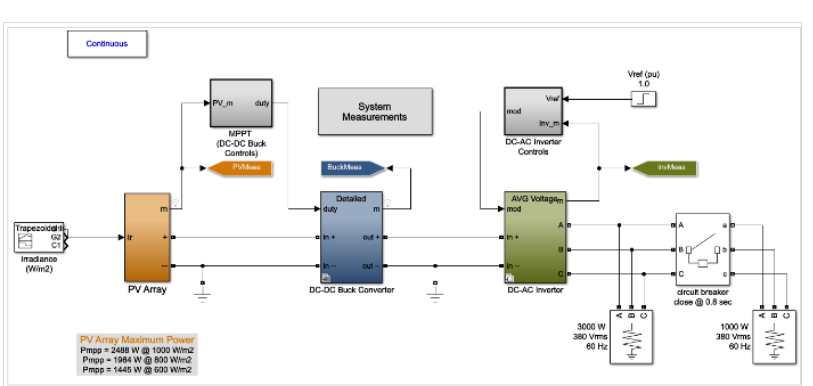

Figure 7. SimuLink model for simulating the MPPT algorithms
For simulating the changing weather conditions, two signals have been used as input to the PV panel: a trapezoidal signal for slowly changing irradiance and a pulse signal for fast changing irradiance, as shown in Figure 8.

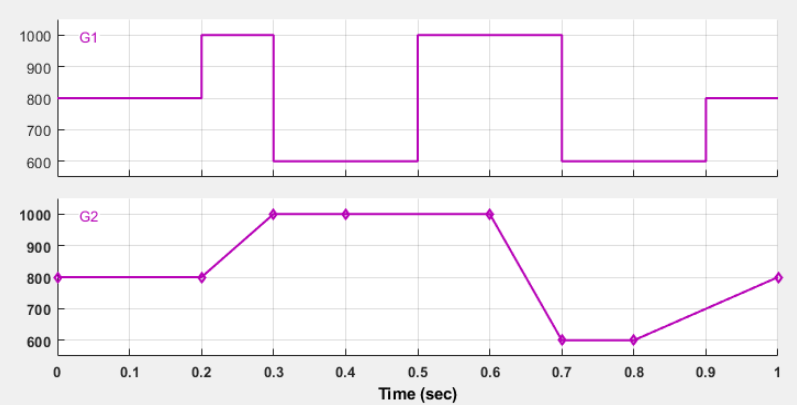

Figure 8. Input signals to the PV array: pulse (G1) and trapezoidal (G2)

In Figure 9 has been plotted the output power of the PV array when stimulated with the two imput signals described above and the MPP being tracked using the standard methods $\mathrm{PnO}$ and INC. As expected, the PnO method is faster, but the amplitude of the oscillations around the Pmax are noticeable.

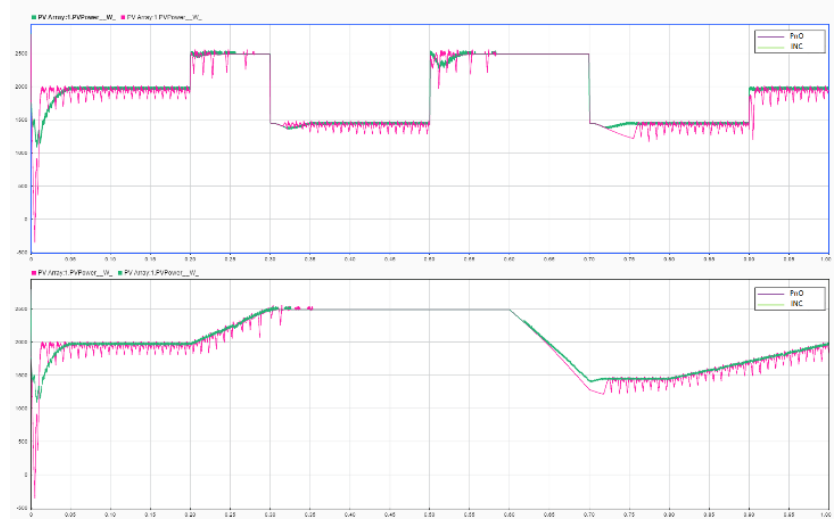

Figure 9. Comparison between $\mathrm{PnO}$ and INC

Both scenarios have been run using $\mathrm{PnO}$ with variable step (PnO-VS) as method for tracking the MPP sand the data has been plotted together with the previous ones in the Figure 10 below.

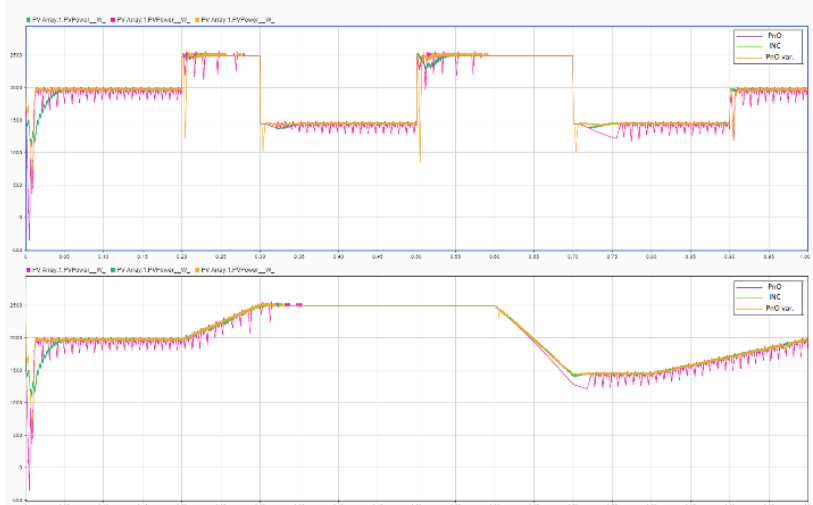

Figure 10. Comparison between PnO-VS, PnO and INC 
The PnO-VS has a response time similar to $\mathrm{PnO}$ standard and the oscillations are limited, similar to the INC algorithm.

As a measure of efficiency, the total power generated over the simulated period of time has been measured and used for comparing the three methods. The results are shown in Table 1.

Table 1 Total Generated Energy

\begin{tabular}{|l|l|l|r|}
\hline & PnO & INC & \multicolumn{1}{c|}{ PnO-VS } \\
\hline Trapezoidal & 1993,56 & 2025,49 & 2032,34 \\
\hline Pulse & 1856,73 & 1887,76 & 1891,55 \\
\hline
\end{tabular}

It's been observed, that in both scenarios the INC algorithm generates about $1,60 \%$ more power than the PnO standard method.

Furthermore, in the pulse scenario, the PnO-VS algorithm generates $0,20 \%$ more power than the INC method and in the trapezoidal scenario $0,34 \%$ more.

\section{CONCLUSION}

This paper presents a modified Perturb and Observe algorithm, which reduces the limitations of classical PnO. By using the PnO-VS algorithm, the trade-off between MPPT response time and power oscillation problem is partly solved.

The simulation results indicate that $\mathrm{PnO}-\mathrm{VS}$ performs better than both $\mathrm{PnO}$ and INC methods. The algorithm does not guarantee, though, that the global MPP would be tracked in case of multiple local maximum power points.

\section{REFERENCES}

[1] Esram, T. and Chapman, P.L., Comparison of Photovoltaic Array Maximum Power Point Tracking Techniques, Energy Conversion, IEEE Transactions on, 22 (43), 2007.

[2] Abouobaida, H. and Cherkaoui, M., Comparative study of Maximum Power Point trackers for fast changing environmental conditions, Multimedia Computing and Systems (ICMCS), 2012.

[3] Challa, D. T. R. And Raghavendar, I., Implementation of Incremental Conductance MPPT with Direct Control Method Using Cuk Converter, International Journal of Modern Engineering Research (IJMER), 2 (4491), 2012.

[4] Elgendy, M. A., Zahawi, B. And Atkinson D.J., Assessment of the Incremental Conductance Maximum Power Point Tracking Algorithm, Sustainable Energy, IEEE Transactions on, 4 (108), 2013.

[5] Bonkoungou, D., Koagala, Z., Njomo, D., Modeling and simulation of photovoltaic module considering single-diode equivalent circuit in Matlab, International Journal of Engineering and Technology and Advanced Engineering, 3 (493), 2013.
[6] El-Khozondar, H.J., El-Khozondar R.J., Matter, K, Suntio, T., A review study of photovoltaic array maximum power tracking algorithms, Springer Open Journal, 2016.

[7] H. Andrei, T. Ivanovici, G. Predusca, E. Diaconu, P.C. Andrei, Curve Fitting Method for Modeling and Analysis of Photovoltaic Cells Characteristics, Automation Quality and Testing Robotics (AQTR), IEEE International Conference on, 2012.

[8] Belkaid, A., Colak I., Kaysli K., Implementation of a Modified P\&O-MPPT Algorithm Adapted for Varying Solar Radiation Conditions, Electrical Engineering, 99 (3), 839, 2017. 